\title{
Statistical Analysis on Diameter Error of Bearing Cylindrical Roller with Different Precision and Levels
}

\author{
Jianbo Wei ${ }^{1}$, Yujun $\mathrm{Xue}^{1,2,3}$, Yongjian $\mathrm{Yu}^{2}$, Jishun $\mathrm{Li}^{2^{*}}$ and Bitao Pang ${ }^{3}$ \\ ${ }^{1}$ School of Mechatronics Engineering, Henan University of Science and Technology, Luoyang \\ 471003 \\ ${ }^{2}$ Henan Key Laboratory for Machinery Design and Transmission System, Henan University of \\ Science and Technology, Luoyang 471003 \\ ${ }^{3}$ State Key Laboratory of Aviation Precision Bearings, Luoyang 471039) \\ * The corresponding author
}

\begin{abstract}
Selected cylindrical roller bearings of type NU204 as the research object, measured diameter of 10 sets of bearing rollers with P0 and P6 precision and level, and accounted respectively statistical data of diameter error of bearing roller of two precision and levels. Made normality test and descriptive analysis on error data of roller diameter, and made comparative study on distribution characteristics of roller diameter error in different levels and precision, then used population of 10 sets of bearings by P0 and P6 precision and level to verify to got normal distribution law. The results showed that about P0-level cylindrical roller bearings, its roller diameter distribution range was wide, but population approximately was matched with normal distribution with big skewness; about P6-level cylindrical roller bearings, its roller diameter distribution range was narrow with small skewness but its height was matched with normal distribution compared with P0-level. Respectively treated roller diameter error data of 10 sets of P0 and P6 precision as sample, overall verified normal distribution law and characters acquired by 4 sets of samples.
\end{abstract}

Keywords: Cylindrical roller bearings; Roller bearings; Errors distribution; Statistical analysis

\section{不同精度等级轴承圆柱滚子直径误差的统计分析}

\author{
魏建波 ${ }^{1}$, 薛玉君 ${ }^{1,2,3}$, 余永健 ${ }^{2}$, 李济顺 ${ }^{2}$, 庞碧涛 ${ }^{3}$ \\ (1. 河南科技大学 机电工程学院 河南洛阳 471003;
}

2. 河南科技大学 河南省机械设计及传动系统重点实验室 河南洛阳 471003

3. 航空精密轴承国家重点实验室 河南洛阳 471039)

摘要: 选取轴承型号为 NU204 的圆柱滚子轴承为研究对象, 测量了 P0 和 P6 精度等级各 10 套轴承滚子的直径, 分别 对两种精度等级的轴承滚子直径误差数据进行了统计, 并对滚子直径误差数据进行了正态性检验和描述性分析, 对比研究了 不同精度等级滚子直径误差的分布特征, 分别用 P0 和 P6 精度等级的 10 套轴承的样本总体对得到的正态分布规律进行了验 证。结果表明: 对于 $\mathrm{P} 0$ 级圆柱滚子轴承, 其滚子直径误差分布区间较宽, 但样本总体近似符合正态分布但偏度较大; 对于 P6 级圆柱滚子轴承, 其滚子直径误差分布区间较 P0 级变窄偏度变小且高度符合正态分布; 分别以 10 套 P0 和 P6 精度等级 的滚子直径误差数据样本总体验证了以 4 套为样本时得到的正态分布规律和特征。

关键词: 圆柱滚子轴承; 轴承滚子; 误差分布; 统计分析

中图分类号: TH16 文献标志码: A

\section{引言}

圆柱滚子轴承广泛应用于精密机床、汽车、航空航天等领域。圆柱滚子轴承的运动精度往往影响到整 
个机械设备的使用寿命、安全性和轴系的运转精度 ${ }^{[1]}$ 。轴承内圈、外圈、滚子等零件的几何误差对轴承运 动精度有着重要影响。为此, 国内外学者开展了许多关于轴承零件几何误差对轴承运动精度影响的相关研 究。陈振强 ${ }^{[2]}$ 在不考虑轴承内、外圈滚道和滚子圆度误差的情况下, 研究了滚子直径误差及滚子排布方式 对轴承外圈径向跳动的影响。武全有 ${ }^{[3]}$ 建立载荷约束下滚子直径尺寸误差与轴承运动精度关系的数学模型, 对圆柱滚子轴承的运动状态进行数值仿真, 研究载荷约束下滚子尺寸误差对轴承运动精度的影响, 得知随 着轴承径向载荷的增大, 内圈径向跳动量呈逐渐减小的趋势, 且逐渐趋于平缓; 多个滚子存在误差时, 内 圈径向跳动量大小与滚子的排布方式有关。吴柏华等 ${ }^{[4]}$ 和李传顺等 ${ }^{[5]}$ 从深沟球轴承内部几何关系入手，考 虑了滚动体的直径误差、内外圈滚道的圆度误差等, 着重分析了滚动体的直径误差以及内外圈滚道圆度误 差对深沟球轴承非重复性跳动的影响规律。王宝坤等 ${ }^{[6]}$ 在考虑轴承内圈转动的情况下, 讨论了滚子直径误 差对轴承径向跳动的影响。Noguchi 等 ${ }^{[7]}$ 从理论上研究了波纹度和滚动体的直径偏差对轴承径向非重复性 跳动的影响规律。 $0 k a m o t o ~{ }^{[8]}$ 通过试验获得球轴承外圈形状精度和球的尺寸差对内圈旋转时轴心的影响, 并 论证了跳动的性质。袁幸和朱永生等 ${ }^{[9]}$ 研究了轴承钢球个数及钢球误差对轴承非重复性跳动影响。

圆柱滚子轴承中的滚子与滚道为线接触, 保证轴承在工作中能够承受较大的径向负荷及高速旋转, 滚 子直径误差是影响轴承运动精度的重要因素 ${ }^{[10,11]}$ 。但是, 目前关于圆柱滚子轴承滚子直径误差分布规律对 轴承旋转精度的影响方面的研究很少见到报道。

本文以圆柱滚子轴承为研究对象, 分别对 P0 和 P6 精度等级的 NU204 型号轴承滚子直径误差进行统计, 对滚子直径误差数据进行正态性检验, 获得 P0 和 P6 精度等级的滚子直径误差分布特征, 为研究轴承滚子 直径误差分布规律对轴承旋转精度的影响提供支持。

\section{1 轴承滚子直径误差的测量和统计}

\section{1 轴承滚子直径误差的测量方法}

使用 Talyrond 圆度仪测量型号为 NU204, 精度等级分别为 P0 和 P6 的圆柱滚子轴承。测量轴承滚子直 径尺寸时, 不能将某一个点位的测量值作为其最终的测量值, 因为不同点位测量值不同; 同时, 也不能将 某个截面测得的测量值作为其最终的测量值, 因为不同截面测量值不同。因此, 每套轴承滚子直径尺寸的 获取都需要测量轴承滚子不同点位、不同截面的尺寸, 然后对所得测量值求平均值, 该平均值就是最终的 测量结果。所测量的点位和截面越多就越能反映轴承滚子直径的真实尺寸。

本试验将滚子从母线方向上四等分, 有三个等分点, 将该所在的点截面作为测量截面, 然后再在截面 上选取三个点, 将测得的值求平均值, 该平均值即为测量最终结果。图 1 是轴承滚道上某截面选取的尺寸 测量点位。a 点位和 $\mathrm{e}$ 点位在同截面上所测所数据相同。

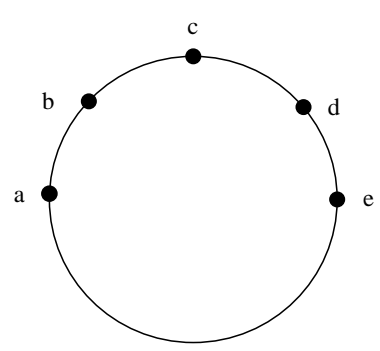

图 1 轴承元件某截面上测量点位 
本文将轴承型号为 NU204, 精度等级为 P0 级和 P6 级的圆柱滚子轴承分别编号为 1-10。轴承滚子直径 误差数据作为样本且文中涉及的直径误差均以 $\mathrm{mm}$ 为单位。将每套轴承滚子每个测量位置数据定义为 $X_{g n}^{k i}$ 分 别求出 $\bar{X}_{g n}^{k i}, \bar{X}_{g n}^{k}, \bar{X}_{g n}$ 。

$\bar{X}_{g n}^{k i} \quad$ 第 $\mathrm{n}$ 套轴承滚子的第 $\mathrm{k}$ 测量平面第 $\mathrm{i}$ 测量点的直径误差均值

$\bar{X}_{g n}^{k}$ 第 $\mathrm{n}$ 套轴承滚子的第 $\mathrm{k}$ 测量平面的直径误差均值

$\bar{X}_{g n}$ 第 $\mathrm{n}$ 套轴承滚子的直径误差均值

其中 $n=1,2, \cdots, 10$ 为轴承的序号; $k=1,2,3$ 为测量平面的序号; $i=1,2, \ldots, 4$ 为测量点的序号。

\section{2 滚子直径误差的统计分析及正态曲线拟合}

首先选取 P0 和 P6 精度等级的 1-4 套轴承进行统计分析, 并检验得到的分布规律是否符合正态分布。 然后分别用 P0 和 P6 精度等级整体的 10 套轴承滚子直径误差数据对得到的正态分布进行检验。

选取试验测得的 NU204P0 级第 1-4 套轴承的滚子数据进行处理得到三组数据矩阵 ${ }^{1 i}, \bar{X}_{g n}^{2 i}, \bar{X}_{g n}^{3 i}$ 其 中 $\mathrm{n}=1,2,3,4 ; \mathrm{i}=1,2,3,4$; 对三组数据的总体样本进行处理并作出正态分布拟合曲线。

从图 2 和图 3 中可以看出, 高精度轴承滚子直径误差的均值和方差都比低精度的轴承更小, 直径误差 的分布更为集中。高精度等时滚子直径误差带呈现区间间断的情况, 分析可能是测量装置的精度受限导致。

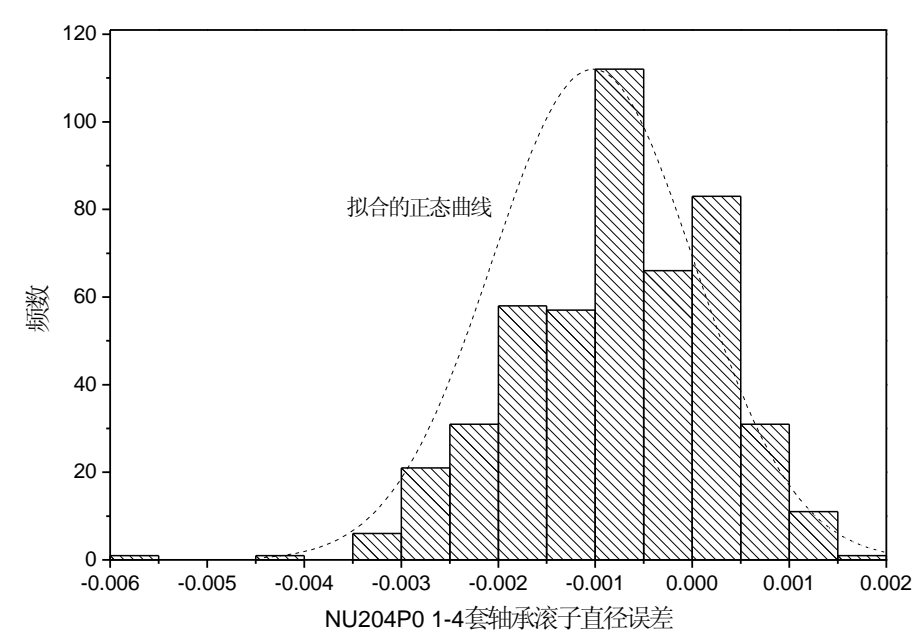

图 2 NU204P0 第 1-4 套轴承滚子样本总体直径误差的频数分布直方图及正态曲线拟合 


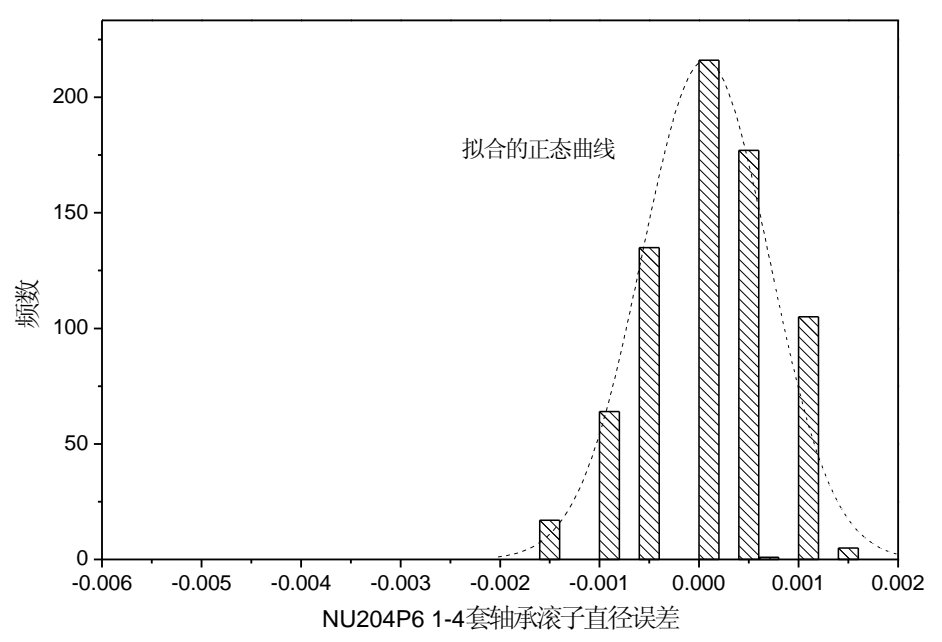

图 3 NU204P6 第 1-4 套轴承滚子样本总体直径误差的频数分布直方图及正态曲线拟合

\section{2 数据的正态性检验及描述性分析}

\section{1 滚子直径误差的正态性检验}

以 NU204P0 级 1-4 套轴承滚子直径误差数据为例进行分析。用 Q-Q 图和 P-P 图判断变量是否服从正态 分布。如果数据服从正态分布，则 $Q-Q$ 图中的数据点应与理论直线（对角线）基本重合。由图 4 知，滚子 直径误差均值的分布与理论分布无明显的差异。图 5 是反映正态分布计算的理论值与实际值之差的分布情 况, 即分布的残差图。 P-P 图如果数据服从正态分布, 则数据的点应比较均匀的分布在 $\mathrm{Y}=0$ 这条直线的上 下方。由图 5 可知, 点的分布比较均匀, 即滚子直径误差均值的分布符合正态分布。

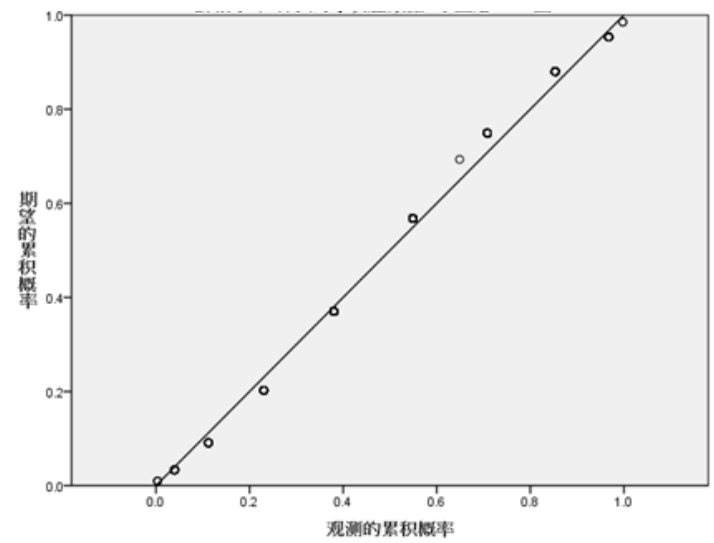

图 4 1-4 套轴承滚子的直径误差数据正态 Q-Q 图 


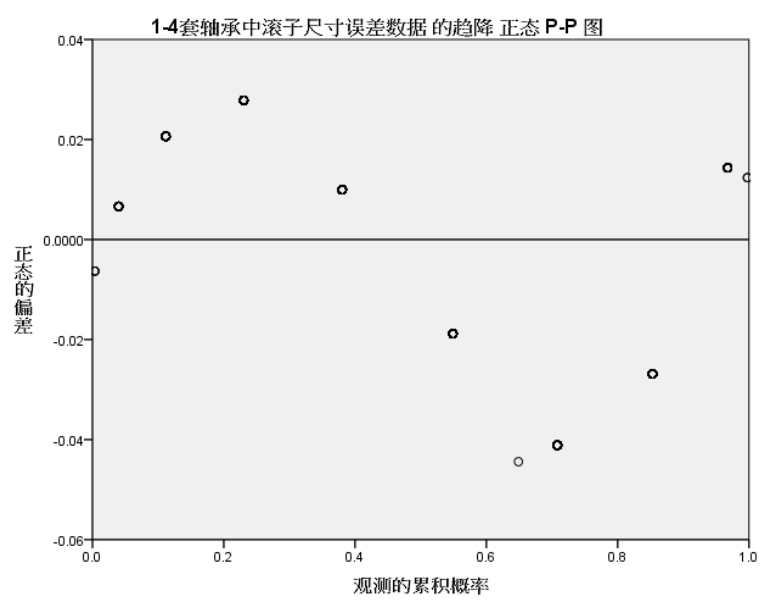

图 5 1-4 套轴承滚子的直径误差数据正态 P-P 图

\section{2 滚子直径误差的描述性分析}

针对轴承滚子直径误差的分布类型问题, 在 SPSS 中可以采用描述性统计的方法对数据有更深入的了 解, 如均值, 标准差, 偏度, 峰度等。偏度和峰度是描述数据分布形态的统计量, 偏度值的为正时, 表示 与标准正态分布相比, 其峰偏向较小的数值方; 其值为负, 则表示与标准正态分布相比, 其峰偏向较大数 值方。峰度值为正时, 表示与标准正态分布相比其分布相对尖锐, 其值为负时, 则表示与正态分布相比其 分布相对平坦。由表 2-1 可知 P6 级滚子直径误差均值、标准差和误差分布范围均比 P0 级的小; P0 和 P6 级滚子直径误差的偏度均为负值, 但 P6 级的偏度绝对值更小; P0 级的滚子直径误差峰度为正, P6 级的峰 度为负，说明精度较高时直径误差的分布更为平坦光滑。

表 2-1 P0 和 P6 级圆柱滚子轴承的描述统计量

\begin{tabular}{ccccccc}
\hline 精度等级 & 轴承套数 & 均值 $(\mathrm{mm})$ & 标准差 $(\mathrm{mm})$ & 偏度 & 峰度 & 误差范围 $(\mathrm{mm})$ \\
\hline \multirow{2}{*}{ P0 } & 4 & -0.001021 & $0.00104 \cdot 29$ & -0.492 & 0.556 & 0.0075 \\
& 10 & -0.000673 & 0.0010367 & -0.277 & 0.441 & 0.0080 \\
\multirow{2}{*}{ P6 } & 4 & 0.000063 & 0.0006400 & -0.263 & -0.452 & 0.0030 \\
& 10 & 0.000206 & 0.0006629 & -0.153 & -0.213 & 0.0035 \\
\hline
\end{tabular}

\section{3 轴承滚子直径误差分布规律验证}

为了验证不同精度等级下, 10 套轴承整体滚子直径误差的分布规律与前面所述一致。作出 P0 和 P6 级 的轴承滚子直径误差的统计分布图进行对比分析： 


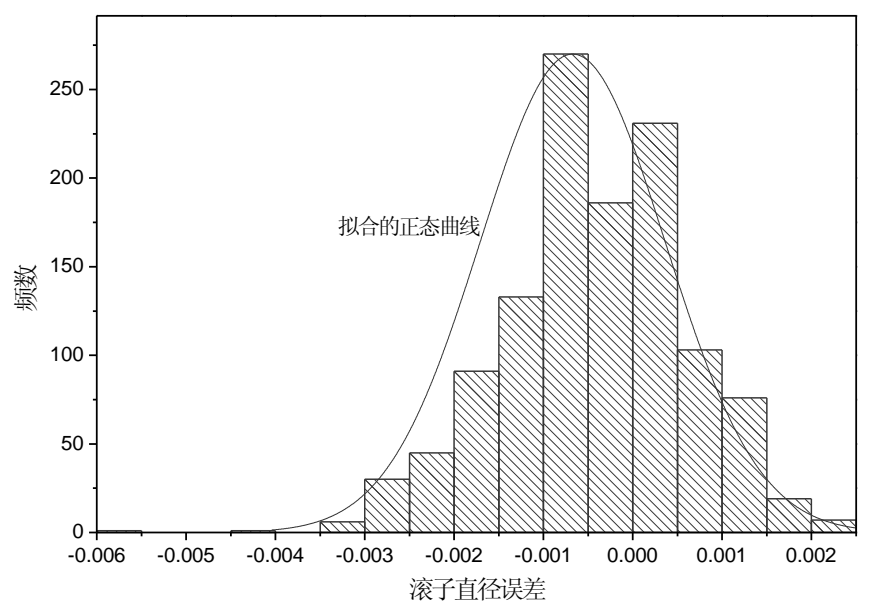

图 6 P0 精度 1-10 套轴承滚子样本总体直径误差分布图

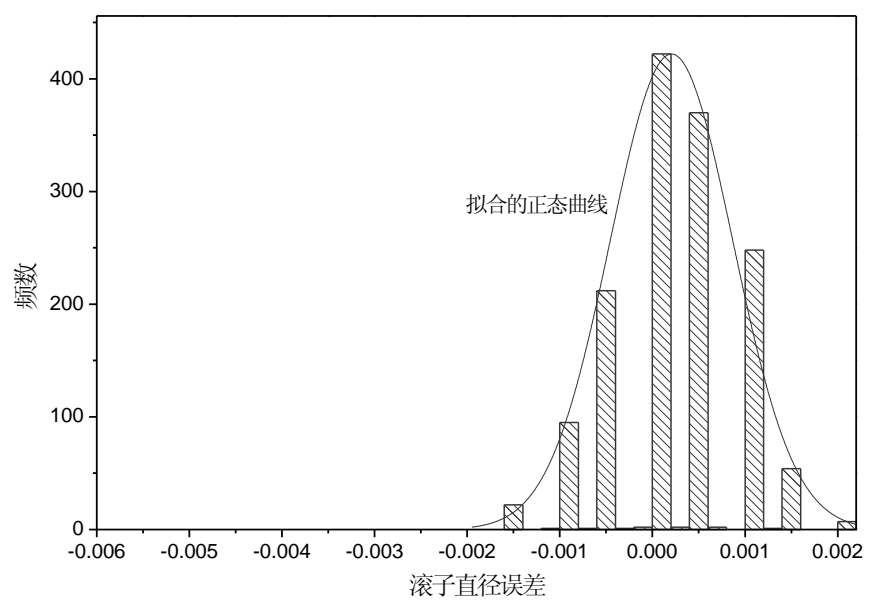

图 7 P6 精度 1-10 套轴承滚子样本总体直径误差分布图

由图 6 和图 7 可以看出 P0 和 P6 精度等级的圆柱滚子轴承滚子的直径误差分布都满足正态分布, 验证 了正态分布拟合圆柱滚子轴承滚子直径误差分布的合理性。

\section{4 结论}

（1）测得了型号为 NU204，精度等级分别为 P0 和 P6 的各 10 套轴承滚子直径。对 P0 和 P6 精度等级 的轴承滚子直径误差数据进行了统计。用正态分布分别拟合了 4 套 P0 和 P6 精度等级的轴承滚子直径误差 数据, 得出其各自的样本总体均符合正态分布。

(2) 以 P0 级 4 套滚子直径误差数据为例, 对其进行了正态性检验。并对 P0 和 P6 精度等级轴承滚子 直径误差数据样本分别为 4 套和 10 套时, 进行了描述性分析。得出了 P6 级滚子直径误差均值、标准差和 误差分布范围均比 P0 级的小; P6 级精度的轴承滚子直径误差相对于 P0 级的偏度绝对值更小, 直径误差的 分布更为集中，拟合的正态曲线更为平坦光滑。

(3) 分别以 10 套 P0 和 P6 精度等级的轴承滚子直径误差数据为样本总体, 验证了以 4 套轴承滚子误 差数据为样本时得到的正态分布规律和特征。 


\section{Acknowledgements}

The authors gratefully thank the National Natural Science Foundation of China (No. 51375148) and the Science Foundation of Henan Province of China (No. 162300410064) for financial support. This work is also supported by the Key Research Program of the Higher Education Institutions of Henan Province (No. 15A460022), and the Program for Innovative Research Team (in Science and Technology) in University of Henan Province (No. 15IRTSTHN008).

\section{参考文献:}

[1] 王红军. 滚动轴承测试技术 $[\mathrm{M}]$. 北京：机械工业出版社，2008：51-54.

[2] 陈振强, 薛玉君, 余永健, 等. 滚子直径误差及排布对轴承径向跳动的影响 $[J]$. 组合机床与自动化加工技术, 2016, 10: 78-82.

[3] 武全有, 薛玉君, 李济顺, 等. 载荷约束下滚子误差对轴承运动精度的影响 [J]. 机械设计与制造, 2016, 01 : $16-19+23$.

[4] 吴柏华, 毛范海, 董惠敏. 深沟球轴承元件几何误差对回转精度的影响 $[J]$. 组合机床与自动化加工技术, 2015 , 01: $22-25$.

[5] 李传顺, 毛范海. 深沟球轴承元件几何误差对非重复性跳动影响 $[J]$. 组合机床与自动化加工技术, 2013, 11: 9-13.

[6] 王宝坤，毛范海，孙守林，等. 圆柱滚子轴承零件几何误差对载荷分布的影响 [J]. 轴承，2014，02：30-35.

[7] NOGUCHI J, HIRUMA K, KAWA H. The influence of location of balls and ball diameter difference in rolling bearings on the nonrepetitive runout (NRRO) of retainer revolution [J]. Tribology International, 2005, 29(1): 11-18.

[8] OKAMOTO J, OHMORI T, KITAHARA T. Study on run-out of ball bearings: relation between unroundness of race and locus of shaft in rotation [J]. Japanese Journal of Tribology. 2001, 46(7): 578-584.

[9] 袁幸, 朱永生, 洪军, 等. 精密球轴承不可重复跳动影响主轴动特性的预测 [J]. 吉林大学学报 (工学版), 2012, $42(2)$ : $382-387$.

[10] 马芳波, 纪鹏, 李正美, 等. 联合载荷下滚子直径误差对球面滚子轴承力学性能的影响 $[\mathrm{J}]$. 中国工程机械学报, 2015, 13(2) : 114-119.

[11] 徐文䩀, 魏泽飞, 孙晶, 等. 轴承滚子电化学机械光整加工表面质量预测与加工参数选择 $[\mathrm{J}]$. 中国机械工程, 2012, 23(5): 525-530.

\section{References:}

[1] Wang Hongjun. Testing Technology in Rolling Bearing [M]. Beijing: China Machine Press, 2008: 51-54.

[2] Chen Zhenqiang, Xue Yujun, Yu Yongjian, etc. Effect of Roller Diameter Error and Arranged on Bearing Radial Runout [J]. Modular Machine Tools \& Automatic Manufacturing Technique, 2016, 10: 78-82.

[3] Wu Quanyou, Xue Yujun, Li Jishun, etc. Effect of Roller Error Under Loading Constraints to Bearing Motion Precision [J]. Machine Design and Manufacture, 2016, 01: 16-19+23.

[4] Wu Baihua, Mao Fanhai, Dong Huimin. Effect of Geometrical error of Deep Groove Ball Bearing Components to Rotary Accuracy [J]. Modular Machine Tools \& Automatic Manufacturing Technique, 2015, 01: 22-25.

[5] Li Chuanshun, Mao Fanhai. Effect of Geometrical error of Deep Groove Ball Bearing Components to Non-repetitive Runout [J]. Modular Machine Tools \& Automatic 
Manufacturing Technique, 2013, 11: 9-13.

[6] Wang Baokun, Mao Fanhai, Sun Shoulin, etc. Effect of Geometrical error of Cylindrical Roller Bearing Parts to Load Distribution [J]. Bearing, 2014, 02: 30-35.

[7] NOGUCHI J, HIRUMA K, KAWA H. The influence of location of balls and ball diameter difference in rolling bearings on the nonrepetitive runout (NRRO) of retainer revolution [J]. Tribology International, 2005, 29(1): 11-18.

[8] OKAMOTO J, OHMORI T, KITAHARA T. Study on run-out of ball bearings: relation between unroundness of race and locus of shaft in rotation [J]. Japanese Journal of Tribology. 2001, 46(7): 578-584.

[9] Yuan Xing, Zhu Yongsheng, Hong June, etc. Prediction that Not Repeatable Runout of Precise Ball Bearing Affect Dynamic Properties of Principal Axis [J]. Journal of Jilin University Engineering and Technology Edition, 2012, 42(2): 382-387.

[10] Ma Fangbo, Ji Peng, Li Zhengmei, etc. Effect of Roller Diameter Error under Combined Loading to Mechanical Property of Spherical Roller Bearing [J]. Chinese Journal of Construction Machinery, 2015, 13(2): 114-119.

[11] Xu Wenji, Wei Zefei, Sun Jing, etc. quality prediction on mechanical skin pass finished surface of bearing roller in electrochemistry [J]. China Machinery Engineering Corporation, 2012, 23(5): 525-530. 